\title{
Efficacy and Toxicity of Treatments for Primary Central System Lymphoma: Review of the Recent Literature
}

\author{
Mohammad Jay ${ }^{1}$, David A. MacDonald ${ }^{2}$ \\ ${ }^{1}$ Faculty of Medicine, University of Ottawa, Ontario, Canada \\ ${ }^{2}$ Department of Hematology, The Ottawa Hospital, Ontario, Canada
}

\section{ABSTRACT}

Primary central nervous system lymphoma (PCNSL) is an uncommon type of central nervous system lymphoma, most commonly presenting as hemiparesis and headache. Currently, there is a wide range of treatments for PCNSL, consisting of various permutations between chemotherapy, radiation and autologous stem cell transplant (ASCT). Although the backbone of PCNSL treatment consists of high-dose methotrexate (HD-MTX), the role of combination versus single agent chemotherapy, combined modality (chemotherapy + radiation) versus chemotherapy or radiation alone, and the use of consolidative ASCT are contested. Surgery does not have a role in the treatment of PCNSL although stereotactic biopsies tend to help with symptomatic relief. Radiation monotherapy is generally reserved for patients with contraindications to chemotherapy or as a palliative measure. Combined chemotherapy and radiation treatment has been shown to have a great efficacy, although its increased neurotoxicity compared to chemotherapy alone is a major drawback. A growing body of research is focused on comparing the efficacy of various chemotherapeutic regimens. Currently, the MATRix regimen comprising of HD-MTX $\left(3.5 \mathrm{~g} / \mathrm{m}^{2}\right)$-cytarabine/rituximab/thiotepa is widely used. The additional survival benefit of ASCT is contested although its role in the treatment of refractory or relapsed PCNSL is generally agreed upon. Finally, intrathecal HD-MTX has been shown to have added survival benefit when added to the standard therapies. Further retrospective and prospective studies are required to compare the efficacy and toxicity of various treatment options, with a focus on different chemotherapeutic agents and ASCT.

\section{RÉSUMÉ}

Le lymphome primitif du système nerveux central (LPSNC) est un type peu commun de lymphome du système nerveux central, qui se présente souvent avec une hémiparésie et une céphalée. Actuellement, il y a une grande variété de traitements pour le LPSNC, consistant en plusieurs permutations de la chimiothérapie, la radiation et l'autogreffe de cellules souches. Bien que le pilier du traitement du LPSNC consiste en méthotrexate à haute dose (MTX-HD), le rôle de multiple versus un seul agent chimiothérapeutique, d'un modèle combiné (chimiothérapie + radiation) versus la chimiothérapie ou la radiation seule, et de l'usage d'autogreffe de cellules souches consolidatrice, sont contestés. La chirurgie n'a pas un rôle dans le traitement du LPSNC malgré que des biopsies stéréotaxiques ont tendance à aider avec un soulagement symptomatique. La radiation comme monothérapie est surtout réservée pour les patients ayant des contrindications à la chimiothérapie ou comme mesure palliative. Le traitement par combinaison de chimiothérapie et de radiation a démontré beaucoup d'efficacité, par contre sa neurotoxicité augmentée comparée à la chimiothérapie seule est un important désavantage. Un montant augmentant de recherche cible la comparaison de l'efficacité d'une variété de régimes chimiothérapeutique. Actuellement, le régime MATRix composé de MTX-HD (3.5 g/m²)/ cytarabine/rituximab/thiotepa est utilisé largement. Les chances de survie augmentée par autogreffe de cellules souches sont contestées, mais son rôle dans le traitement du LPSNC réfractaire ou récurrent est généralement convenu. Finalement, le MTXHD intrathécal a démontré des avantages pour la survie, quand ajouter aux thérapies standards. Des études rétrospectives et prospectives sont requises pour comparer l'efficacité et la toxicité de la variété d'options de traitement, avec une cible sur les différents agents chimiothérapeutiques et l'autogreffe de cellules souches.

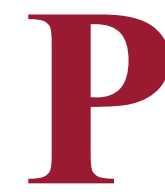

rimary central system lymphoma (PCNSL) is an uncommon type of non-Hodgkin lymphoma, comprising $3-4 \%$ of brain tumours and $4-6 \%$ of extranodal lymphomas $(1,2)$. Its incidence is decreasing in the general population, but is increasing in those above the age of 65 (3).

Acquired or genetic immunodeficiency are the established

Keywords: Primary Central Nervous System Lymphoma; Chemotherapy; Radiation Therapy 
risk factors for developing PCNSL. Specifically, patients with human immunodeficiency virus (HIV) and congenital immunodeficiency disorders (e.g. Wiskott-Aldrich syndrome) carry a risk of $2-6 \%$ and $4 \%$ compared to the general population for developing PCNSL, respectively. Other diseases inducing immunosuppression (e.g. systemic lupus erythematosus) and post-transplant status have also been associated with increased risk of PCNSL $(3,4)$.

The international Extranodal Lymphoma Group suggest using age (more or less than 60), Eastern Cooperative Oncology Group(ECOG) performance status (0-1 versus 2-4), serum lactase dehydrogenase level (normal versus elevated), cerebrospinal fluid (CSS) protein concentration (normal versus elevated), and involvement of deep brain structures (no versus yes) as prognostic indicators for PCNSL (5). Unfortunately, the prognosis of PCNSL remains poor despite the emergence of new therapies in the field (6).

The clinical signs and symptoms vary depending on the site of involvement in the central nervous system. PCNSL most commonly involves the periventricular white matter (51\%) and basal ganglia (48.9\%). The most frequent presenting symptoms were shown to be hemiparesis $(56.2 \%)$ and headache $(51.7 \%)$ in a retrospective study of 176 patients with PCNSL (7). Other common symptoms include neuropsychiatric symptoms and other signs of raised intracranial pressure (i.e. nausea and vomiting). Seizure and visual symptoms occur less frequently in $14 \%$ and $4 \%$ of patients, respectively (8).

Suspicion of PCNSL should prompt imaging of the central nervous system, with MRI as the preferred modality. Subsequent investigations include lumbar puncture for CSF analysis, slit lamp examination of both eyes for potential ocular pathology and stereotactic needle biopsy of the involved tissue (9). The definitive diagnosis of PCNSL is made by histopathological analysis. Therefore, brain biopsy should not be delayed after the suspicion of PCNSL (9). Once the diagnosis of PCNSL is made, a whole-body PET scan and bone marrow biopsy should be performed to rule out secondary diseases with CNS involvement (10).

Here, we review the existing literature on studies comparing the effectiveness and toxicity of various modalities in the treatment of PCNSL. We also attempt to appraise and identify the existing gaps in the literature.

\section{TREATMENT OVERVIEW}

Treatment of PCNSL consists of an induction and a consolidation phase. The induction phase aims at achieving a complete radiographic response (CR), while the goal of consolidation phase is to maintain remission. It is commonly accepted that high-dose $\left(3-8 \mathrm{~g} / \mathrm{m}^{2}\right)$ methotrexate (HD-MTX) is the backbone of PCNSL induction therapy. The roles of whole brain radiation, combined versus single agent chemotherapy and autologous stem cell transplant in both phases are debated. As such, there is no agreed upon algorithm in the treatment of PCNSL (11). Such lack of consensus stems from the rarity of the disease and lack of sufficient large scale randomized clinical trials (9). Generally, PCNSL is managed by a multidisciplinary team including hematologists, radiation oncologists, neuroradiologic, neurosurgeons, ophthalmologists, and allied health care professionals $(12,13)$.

\section{SURGERY}

Currently, there is no role for surgery in the treatment of PCNSL. The tumour is usually deeply located in the brain, making surgical access difficult. Also, the multifocal and diffuse nature of the disease allows for microscopic infiltration across the visible margins of the tumour (14). The data from a retrospective study of 33 patients suggested that while surgery does not improve survival outcomes, it does not play a negative role in survival (15). The authors argued that microsurgical excision of tumours with a single focus and progressive neurological deterioration can improve survival (15). Further studies involving larger groups of patients are required to further explore the exact role of surgery in the treatment of PCNSL.

\section{RADIATION MONOTHERAPY}

Due to its diffuse nature, radiotherapy of PCNSL should involve the whole brain. Up until 1980, whole brain radiotherapy (WBRT) monotherapy was the mainstay of therapy for PCNSL (9). Unfortunately, the outcomes of this treatment were shown to be poor with 5 year-survival of less than 10\% (16). One of the most promising results of WBRT monotherapy was demonstrated in a study led by the Radiation Therapy Oncology Group, where $62 \%$ of patients achieved CR. Unfortunately, the response was not durable with only $48 \%$ and $28 \%$ survival after 1 year and 2 years, respectively (17). Currently, the use of WBRT is recommended in patients with contraindications to chemotherapy and those failing to achieve CR following systemic chemotherapy (18). 


\section{COMBINED RADIOTHERAPY AND CHEMOTHERAPY}

To increase the response duration, different chemotherapeutic agents were added to WBRT. The RTOG conducted a trial in which 54 patients received two or three cycles of cyclophosphamide, doxorubicin, vincristine and dexamethasone (CHOD), followed by high-dose WBRT (total dose of $59.4 \mathrm{~Gy}$ ). The 2 -year survival was $42 \%$ and the median survival for the entire group was 16.1 months. Compared to the study above by Nelson et al. (1992), which investigated radiation monotherapy, addition of CHOD to WBRT did not lead to a significant improvement in overall survival (OS) (19). This lack of efficacy is attributed to poor permeability of blood brain barrier (BBB) to vincristine and doxorubicin (13).

Addition of HD-MTX to WBRT in subsequent studies was shown to be effective. Namely, upfront treatment of 52 patients with HD-MTX $\left(3.5 \mathrm{~g} / \mathrm{m}^{2}\right)$, procarbazine, vincristine and intrathecal methotrexate (MTX), followed by a consolidation therapy with high-dose WBRT (45 Gy) and high dose-cytarabine led to a remarkable improvement in OS compared to previous studies. The mean OS was 60 months with relapse occurring in 18 patients after 3-35 months. Interestingly, the OS of the elderly population was the same with or without WBRT, although addition of WBRT led to higher rates of late neurotoxicity in this population (20). Subsequent studies produced similar results, confirming that the combination of HD-MTX plus WBRT, is superior to WBRT alone (21-23).

So far, there is only one prospective phase 3 randomized clinical trial which investigated the difference between first line HD-MTX $\left(4 \mathrm{~g} / \mathrm{m}^{2}\right)$ combined with WBRT (45 Gy) as compared to chemotherapy alone. The objective of the study was to demonstrate that HD-MTX treatment is non-inferior to HD-MTX and WBRT, with a margin of 0.9. Although the OS was not significantly different between the two arms (32.4 months in HD-MTX/WBRT, confidence interval: 25.8-39.0; versus 37.1 months in HD-MTX alone, confidence interval: 27.5-46.7), the progression free survival (PFS) was higher in the arm receiving WBRT (18.3 months in HD-MTX/WBRT; 11.9 months in HD-MTX alone). The non-inferiority hypothesis of the study was not met as the confidence interval crossed the 0.9 margin set initially. Finally, the patients receiving WBRT experienced greater rates of neurotoxicity (24). Despite its interesting findings, a variety of methodological issues existed in the study, calling the validity of its conclusions into question. Specifically, from the 551 patients enrolled, 411 met the eligibility criteria for the intention to treat group and only 318 patients were treated per protocol due to 93 protocol violations. Once the 93 patients, in whom the protocol violations were committed, were excluded from the denominator, a source of bias was introduced as many of those excluded failed to achieve $C R$ and therefore had a lower PFS (25).

Nowadays, WBRT is mainly used as consolidation treatments. Its major downside is the side effect of neurotoxicity, which is more pronounced when WBRT is used in high doses or in combination with chemotherapy. As a result of this toxicity, many centres avoid using WBRT (13). To account for the issue of neurotoxicity, a few recent studies have investigated the effect of dose-reduced WBRT (dR-WBRT). In a single centred study, the induction therapy consisted of rituximab, MTX, procarbazine, and vincristine. The patients attaining $C R(2 / 3$ of the study population in this case) were then treated with $\mathrm{dR}$ WBRT (23.4 Gy), followed by a consolidation treatment with cytarabine. The results were unparalleled with a median PFS of 7.7 years, 3-year OS of $87 \%$, and stable neuropsychological testing scores 48 months following treatment in those completing the regimen. This study was also unique for using immunotherapy in the treatment of PCNSL (26). An ongoing trial is exploring the role of immunotherapy alone by comparing PFS in a group treated with immunotherapy and WBRT, versus immunotherapy alone (NCT01399372).

\section{SYSTEMIC CHEMOTHERAPY}

Intravenous HD-MTX administered as rapid infusion is the most effective agent in the treatment of PCNSL. Depending on the centre, HD-MTX is administered alone or in combination with other medications. A retrospective study of 288 immunocompetent patients demonstrated that doses of MTX above $3 \mathrm{~g} / \mathrm{m}^{2}$ led to improved survival (27). The best therapeutic dose of HD-MTX is currently not agreed upon, however. To compare the effectiveness and toxicity of HDMTX monotherapy versus HD-MTX combined with other chemotherapeutic agents, a phase II randomized trial was conducted. The authors concluded that HD-MTX combination with cytarabine led to an increase in PFS and a better CR rate compared to HD-MTX monotherapy. Finally, treatment-related toxicity was higher in the polychemotherapy group (28).

Prospective studies exploring HD-MTX monotherapy (usually $8 \mathrm{~g} / \mathrm{m}^{2}$ ) demonstrated a 2-year OS of $61-63 \%$, while those investigating the role of HD-MTX combination therapy revealed a greater 2-year OS of $65-78 \%(13,29$, 30). In terms of combination therapy, rituximab is most commonly combined with HD-MTX. The superiority of HDMTX-rituximab (HD-MTX/R) combination over HD-MTX 
monotherapy was demonstrated in a retrospective study. The results demonstrated an overall improvement in OS and PFS. Remarkably, the median PFS improved from 4.5 months in patients treated with HD-MTX alone to 26.7 in those treated with HD-MTX/R (31). Subsequently, a phase II study of patients aged 18-70 years demonstrated that the MATRix regimen was superior to HD-MTX-cytarabine, and HD-MTX-cytarabine/ thiotepa (32). The CRs after 30 months in the HD-MTXcytarabine/rituximab/thiotepa, HD-MTX-cytarabine/rituximab, and MTX-cytarabine were $49 \%, 30 \%$, and $23 \%$, respectively. The authors of this study concluded that the MATRix regimen can be used as a new chemoimmunotherapeutic regimen in the treatment of PCNSL in patients under the age of 70 . Unfortunately, grade 4 hematological toxicity (i.e. neutropenia and thrombocytopenia) were higher in patients treated with the MATRix regimen as compared with the other two groups (32).

\section{HIGH-DOSE CHEMOTHERAPY/AUTOLOGOUS STEM CELL} TRANSPLANTATION (HDC/ASCT)

HDC/ASCT seems to be a reasonable option for relapsed/ refractory PCNSL. A single-arm multicentre study used an induction regimen of rituximab, high-dose cytarabine and thiotepa, followed by HDC/ASCT conditioning of rituximab, carmustine and thiotepa to test the effectiveness of HDC/ ASCT on immunocompetent patients ( $<66$ years) who were refractory to HD-MTX-based regimens. Patients received HDC/ASCT regardless of their response to induction therapy. Those not achieving CR following HDC/ASCT were treated with WBRT. The results following HDC/ASCT were remarkable for CR of $56.4 \%$, 2-year PFS of $46.0 \%$ and OS rates of $56.4 \%$. Unfortunately, 4 treatment-related deaths were reported (33). This study confirmed similar conclusions by earlier studies regarding the effectiveness of HDC/ASCT in the treatment of refractory/relapsed PCNSL. Despite the promising responses, the restrictive age criteria introduced a selection bias.

Another study expanded the inclusion criteria of the previous study, looking at relapsed and refractory diseases as well as those with partial response to first-line therapy. Patients were treated with high-dose cytarabine and etoposide as salvage treatment, followed by intensive combined thiotepa, busulfan, cyclophosphamide and ASCT. The 2-year OS was $45 \%$ in all patients and $69 \%$ amongst those who completed the treatment. Also, the 2-year PFS was $43 \%$ in the entire population and $58 \%$ in the HDC/ASCT subpopulation (34). The promising results of these studies allowed certain guidelines to recommend HDC/ASCT as an option for chemotherapy- sensitive patients with relapsed or refractory PCNSL (35).

The role of HDC/ASCT as first line therapy for PCNSL has also been investigated. A study used HD-MTX $\left(8 \mathrm{~g} / \mathrm{m}^{2}\right)$, cytarabine and thiotepa as induction therapy, followed by carmustine and thiotepa as conditioning therapy. The patients were subsequently treated by ASCT. Promising results were found with a 5 -year OS of $69 \%$ for all patients and $87 \%$ for patients completing the entire regimen. All of the patients receiving $\mathrm{HDC}$ experienced $\mathrm{WHO}$ grade $3 / 4$ neutropenia and thrombocytopenia. Also, $16.7 \%$ of the patients experienced leukoencephalopathy after a median follow-up of 63 months. Regardless of the neurotoxicity, the authors considered the treatment effective and this amount of toxicity minimal (36).

A centre in Canada reproduced similar results with a different combination of upfront thiotepa, busulfan, cyclophosphamide, and ASCT. Although the 5-year OS was lower (44\%) than that reported by Illerhaus et al. (2006), no neurotoxicity was observed. The choice of medications in this study is remarkable as both busulfan and thiotepa penetrate the BBB at levels greater than $90 \%$, while other common agents, such as carmustine, cyclophosphamide and etoposide have much lower penetration levels (15-70\%, 20\% and 5\%, respectively). Moreover, both busulfan and thiotepa have steep doseresponse curves, further allowing high concentration in the CNS. The unique pharmacokinetics suggests that busulfan and thiotepa have greater potency compared to other medications, while resulting in a lower side effect profile. Furthermore, the treatment-related-mortality (TRM) was relatively high (14\%). Notably, all of the mortalities were observed in patients who were over the age of 60 and had poor performance status (37).

To summarize, HDC/ASCT presents a promising treatment for patients with refractory and relapsed PCNSL. Although various studies have demonstrated the effectiveness of HDC/ ASCT as first-line therapy, no study to date has compared HDC/ASCT, HD-MTX-based chemotherapies or combination chemoradiotherapy as first line therapies. Two ongoing clinical trials attempt to compare the efficacy and toxicity of HDC/ASCT versus chemotherapy or WBRT (NCT01011920 and NCT00863460). In general, HDC/ASCT seems to have a greater benefit to harm ratio in immunocompetent patients who are under the age of 60 and have good performance status (KPS $>60 \%$ at the time of transplant). As such, this population should be the primary target of HDC/ASCT until further data on the use of HDC/ASCT in PCNSL emerges $(13,37)$. 


\section{CEREBROSPINAL FLUID THERAPIES}

The exact role of intrathecal (IT) chemotherapy is controversial, partly due to the paucity of evidence in the literature around its effectiveness. Three retrospective studies demonstrated no benefit in terms of disease control, survival and neurotoxicity with IT therapies comprised mainly of HD-MTX in the treatment of PCNSL (38-40). One out of the three studies specifically explored the role of prophylactic HD-MTX-based IT therapies (38).

On the other hand, a prospective phase II study in 2009 demonstrated promising results with the use of IT therapies (41). Prior to this study, Pels et al. (2003) conducted another phase II study where they used chemotherapeutic regimens based on HD-MTX and HD-cytarabine, in combination with intraventricular MTX, prednisone and cytarabine. The results were promising with a $\mathrm{CR}$ rate of $61 \%$, median OS of 50 months, and median time to treatment failure (TTF) of 15 months (42). The study in 2009 aimed to explore the role of IT therapy by using the same regimen without IT therapy. While the $C R$ rate was comparable (53\%) to the previous study, the rate of early relapse was much higher with a TTF of only 8 months (41). Put together, the two studies demonstrated a clear benefit in using direct chemotherapy injection into the CSF, which is in contradiction to the conclusion by previous retrospective studies. No significant neurotoxicity was observed in either study.

\section{ELDERLY PATIENTS}

Although the role of HD-MTX in the treatment of PCNSL in the elderly is well established, there is insufficient evidence comparing the effectiveness and toxicity of other agents combined with HD-MTX. The only randomized control study comparing MTX-based chemotherapies in the elderly ( $>60$ years) was a phase II trial comparing MTX and temozolomide combination (MT arm) versus MTX, procarbazine, vincristine, followed by consolidation with cytarabine (MPV-A arm). Treatment in the MPV-A arm was associated with enhanced OS, PFS and CR compared to the MT arm. Toxicity was similar between the two arms, with abnormalities in liver function tests as the most common manifestations of toxicity (43). Neuropsychological testing did not detect any neurotoxicity in either group and EORTC QLQ-BN20/QLQ-C30 questioners demonstrated the quality of life was enhanced in both groups. Further details about dosing, efficacy, and toxicity of MTX/ temozolomide and MTX/procarbazine/vincristine/cytarabine regimens are being investigated in an ongoing clinical trial (NCT00503594) (20).

WBRT does not seem to be a reasonable choice in the elderly population due to its disproportionately higher risk to benefit ratio. A systematic review exploring the effects of various first line therapies in the treatment of PCNSL in elderly patients $(\geq 60)$ reported that although WBRT leads to a slight increase in PFS and OS, it is associated with unacceptable increase in neurotoxicity (1). Two retrospective studies demonstrated similar results in terms of neurotoxicity with no change in OS as a result of treatment with WBRT $(20,44)$.

In general, treatments based on HD-MTX are well tolerated and enhance the quality of life in elderly patients. No difference seems to exist between treatment with HD-MTX plus an oral agent, versus HD-MTX plus more aggressive IV therapies. As such, combination of HD-MTX and oral agents (e.g. procarbazine) is preferred (1).

\section{CONCLUSION}

Over the past years, a growing amount of literature has described the characteristics and novel treatments for PCNSL. HD-MTX is the backbone of therapy in the treatment of PCNSL, with HD-MTX-based combination therapies producing better survival outcomes than HD-MTX alone. Similarly, combination of WBRT and HD-MTX-based chemotherapies has been shown to lead to greater survival outcomes than chemotherapy alone. Unfortunately, HD-MTX-based combination chemotherapy and combined chemoradiation are associated with greater toxicity compared to HD-MTX alone and chemotherapy alone, respectively. Therefore, the risks and benefits of various therapies (including the ongoing clinical trials) should be clearly discussed with patients and the choice of therapy should be considered in relation to the patient's goal of care and available resources. Finally, HDC/ASCT seems to be an appropriate option for refractory/relapsing PCNSL. Despite the advances in the treatment of PCNSL, the prognosis of this disease remains poor and many questions regarding the best approaches to its therapy, particularly in the elderly population, remain unanswered.

\section{FUTURE DIRECTIONS}

Further retrospective and prospective studies are required to explore the ideal therapies for various patient populations. Given the promising results of the study showing the superiority 
of the combination of immunotherapy and dR-WBRT, further studies should investigate the role of immunotherapy in PCNSL. Finally, the upfront use of ASCT in combination with chemotherapy and/or radiation should be further elucidated. Retrospective studies can also determine clinicians' tendency for using ASCT in elderly versus younger patients

\section{REFERENCES}

1. Kasenda B, Ferreri AJ, Marturano E, Forst D, Bromberg J, Ghesquieres H, et al. First-line treatment and outcome of elderly patients with primary central nervous system lymphoma (PCNSL) - a systematic review and individual patient data meta-analysis. Ann Oncol. 2015;26(7):1305-13.

2. Villano J, Koshy M, Shaikh H, Dolecek T, McCarthy B. Age, gender, and racial differences in incidence and survival in primary CNS lymphoma. Br J Cancer. 2011;105(9):1414.

3. Krough-Jensen M, Amore F, Jensen M, Christensen B, Thorling K, Pedersen $M$, et al. Clinicopathological Features, Survival And Prognostic Factors Of Primary Central Nervous System Lymphomas: Trends in Incidence of Primary Central Nervous System Lymphomas and Primary Malignant Brain Tumors in A Well-Defined Geographical Area; Population-Based Data from the Danish Lymphoma Registry, Lyfo, And the Danish Cancer Reistry. Leuk Lymphoma. 1995;19(3-4):223-33.

4. Schabet M. Epidemiology of primary CNS lymphoma. J Neurooncol. 1999;43(3):199-201.

5. Ferreri AJ, Blay J-Y, Reni M, Pasini F, Spina M, Ambrosetti A, et al. Prognostic scoring system for primary CNS lymphomas: the International Extranodal Lymphoma Study Group experience. J Clin Oncol. 2003;21(2):266-72.

6. DeFilipp Z, Li S, El-Jawahri A, Armand P, Nayak L, Wang N, et al. High-dose chemotherapy with thiotepa, busulfan, and cyclophosphamide and autologous stem cell transplantation for patients with primary central nervous system lymphoma in first complete remission. Cancer. 2017;123(16):3073 9.

7. Ashrafi F, Zali A, Amiri M, Shabani F. Clinical Presentations of 176 Cases of Primary Central Nervous System Lymphoma: A Case Series. Archives of Neuroscience. 2016;3(2).

8. Bataille B, Delwail V, Menet E, Vandermarcq P, Ingrand P, Wager M, et al. Primary intracerebral malignant lymphoma: report of 248 cases. J Neurosurg. 2000;92(2):261-6.

9. Schaff LR, Grommes C. Updates on Primary Central Nervous System Lymphoma. Curr Oncol Rep. 2018;20(2):11.

10. Abrey LE, Batchelor TT, Ferreri AJ, Gospodarowicz M, Pulczynski EJ, Zucca $E$, et al. Report of an international workshop to standardize baseline evaluation and response criteria for primary CNS lymphoma. J Clin Oncol. 2005;23(22):5034-43.

11. Tinmouth J, Kennedy E, Baron D, Burke M, Feinberg S, Gould M, et al. A Quality Initiative of the Program in Evidence-Based Care (PEBC), Cancer Care Ontario (CCO) Guideline for Colonoscopy Quality Assurance in Ontario. A Quality Initiative of the Program in Evidence-Based Care (PEBC), Cancer Care Ontario (CCO). 2014;28(5):21.

12. Hoang-Xuan K, Bessell E, Bromberg J, Hottinger AF, Preusser M, Rudà R, et al. Diagnosis and treatment of primary CNS lymphoma in immunocompetent patients: guidelines from the European Association for NeuroOncology. The Lancet Oncology. 2015;16(7):e322-e32. doi: https://doi. org/10.1016/S1470-2045(15)00076-5.

13. Kerbauy MN, Moraes FY, Lok BH, Ma J, Kerbauy LN, Spratt DE, et al. Challenges and opportunities in primary CNS lymphoma: A systematic review. Radiother Oncol. 2017;122(3):352-61.

14. Lai R, Rosenblum MK, DeAngelis LM. Primary CNS lymphoma A wholebrain disease? Neurology. 2002;59(10):1557-62.

15. Bellinzona M, Roser F, Ostertag H, Gaab R, Saini M. Surgical removal of primary central nervous system lymphomas (PCNSL) presenting as space occupying lesions: a series of 33 cases. Eur J Surg Oncol. 2005;31(1):100-5.

16. Shibamoto Y, Ogino H, Hasegawa M, Suzuki K, Nishio M, Fujii T, et al. Re- sults of radiation monotherapy for primary central nervous system lymphoma in the 1990s. International Journal of Radiation Oncology• Biology• Physics. 2005;62(3):809-13.

17. Nelson DF, Martz KL, Bonner H, Nelson JS, Newall J, Kerman HD, et al. NonHodgkin's lymphoma of the brain: can high dose, large volume radiation therapy improve survival? Report on a prospective trial by the Radiation Therapy Oncology Group (RTOG): RTOG 8315. International Journal of Radiation Oncology* Biology* Physics. 1992;23(1):9-17.

18. Citterio G, María Ferreri AJ, Reni M. Current uses of radiation therapy in patients with primary CNS lymphoma. Expert Rev Anticancer Ther. 2013;13(11):1327-37. doi: 10.1586/14737140.2013.851007.

19. Schultz C, Scott C, Sherman W, Donahue B, Fields J, Murray K, et al. Preirradiation chemotherapy with cyclophosphamide, doxorubicin, vincristine, and dexamethasone for primary CNS lymphomas: initial report of radiation therapy oncology group protocol 88-06. J Clin Oncol. 1996;14(2):55664. doi: 10.1200/jco.1996.14.2.556. PubMed PMID: 8636771.

20. Abrey LE, Yahalom J, DeAngelis LM. Treatment for primary CNS lymphoma: the next step. J Clin Oncol. 2000;18(17):3144-50.

21. Poortmans PMP, Kluin-Nelemans HC, Haaxma-Reiche $H$, Veer MVt, Hansen M, Soubeyran P, et al. High-Dose Methotrexate-Based Chemotherapy Followed by Consolidating Radiotherapy in Non-AIDS-Related Primary Central Nervous System Lymphoma: European Organization for Research and Treatment of Cancer Lymphoma Group Phase II Trial 20962. J Clin Oncol. 2003;21(24):4483-8. doi: 10.1200/jco.2003.03.108. PubMed PMID: 14597741.

22. DeAngelis LM, Seiferheld W, Schold SC, Fisher B, Schultz CJ. Combination Chemotherapy and Radiotherapy for Primary Central Nervous System Lymphoma: Radiation Therapy Oncology Group Study 93-10. J Clin Oncol. 2002;20(24):4643-8. doi: 10.1200/jco.2002.11.013. PubMed PMID: 12488408.

23. Gavrilovic IT, Hormigo A, Yahalom J, DeAngelis LM, Abrey LE. Long-Term Follow-Up of High-Dose Methotrexate-Based Therapy With and Without Whole Brain Irradiation for Newly Diagnosed Primary CNS Lymphoma. J Clin Oncol. 2006;24(28):4570-4. doi: 10.1200/jco.2006.06.6910. PubMed PMID: 17008697.

24. Thiel E, Korfel A, Martus P, Kanz L, Griesinger F, Rauch M, et al. High-dose methotrexate with or without whole brain radiotherapy for primary CNS lymphoma (G-PCNSL-SG-1): a phase 3, randomised, non-inferiority trial. The lancet oncology. 2010;11(11):1036-47.

25. Cabanillas F. How important is whole brain radiotherapy for treatment of primary CNS lymphoma? The Lancet Oncology. 2010;11(11):1011-2. doi: 10.1016/S1470-2045(10)70234-5.

26. Morris PG, Correa DD, Yahalom J, Raizer JJ, Schiff D, Grant B, et al. Rituximab, methotrexate, procarbazine, and vincristine followed by consolidation reduced-dose whole-brain radiotherapy and cytarabine in newly diagnosed primary CNS lymphoma: final results and long-term outcome. Journal of clinical oncology: official journal of the American Society of Clinical Oncology. 2013;31(31):3971-9.

27. Reni M, Ferreri AJ, Guha-Thakurta N, Blay J-Y, Dell'Oro S, Biron P, et al. Clinical relevance of consolidation radiotherapy and other main therapeutic issues in primary central nervous system lymphomas treated with upfront high-dose methotrexate. International Journal of Radiation Oncology• Biology• Physics. 2001;51(2):419-25.

28. Ferreri AJ, Reni M, Foppoli M, Martelli M, Pangalis GA, Frezzato M, et al. High-dose cytarabine plus high-dose methotrexate versus high-dose methotrexate alone in patients with primary CNS lymphoma: a randomised phase 2 trial. Lancet. 2009;374(9700):1512-20. Epub 2009/09/22. doi: 10.1016/s0140-6736(09)61416-1. PubMed PMID: 19767089.

29. Guha-Thakurta N, Damek D, Pollack C, Hochberg FH. Intravenous methotrexate as initial treatment for primary central nervous system lymphoma: response to therapy and quality of life of patients. J Neurooncol. 1999;43(3):259-68.

30. Cheng $A L$, Yeh $\mathrm{KH}$, Uen WC, Hung RL, Liu MY, Wang $\mathrm{CH}$. Systemic chemotherapy alone for patients with non-acquired immunodeficiency syndrome-related central nervous system lymphoma. Cancer. 
1998;82(10):1946-51.

31. Holdhoff M, Ambady P, Abdelaziz A, Sarai G, Bonekamp D, Blakeley J, et al. High-dose methotrexate with or without rituximab in newly diagnosed primary CNS lymphoma. Neurology. 2014;83(3):235-9.

32. Ferreri AJ, Cwynarski K, Pulczynski E, Ponzoni M, Deckert M, Politi LS, et al. Chemoimmunotherapy with methotrexate, cytarabine, thiotepa, and rituximab (MATRix regimen) in patients with primary CNS lymphoma: results of the first randomisation of the International Extranodal Lymphoma Study Group-32 (IELSG32) phase 2 trial. The Lancet Haematology. 2016;3(5):e217-e27.

33. Kasenda B, Ihorst G, Schroers R, Korfel A, Schmidt-Wolf I, Egerer G, et al. High-dose chemotherapy with autologous haematopoietic stem cell support for relapsed or refractory primary CNS lymphoma: a prospective multicentre trial by the German Cooperative PCNSL study group. Leukemia. 2017;31(12):2623.

34. Soussain C, Hoang-Xuan K, Taillandier L, Fourme E, Choquet S, Witz F, et al. Intensive chemotherapy followed by hematopoietic stem-cell rescue for refractory and recurrent primary CNS and intraocular lymphoma: Societe Francaise de Greffe de Moelle Osseuse-Therapie Cellulaire. J Clin Oncol. 2008;26(15):2512-8.

35. Hoang-Xuan K, Bessell E, Bromberg J, Hottinger A, Preusser M, Rudà R, et al. European Association for Neuro-Oncology Task Force on Primary CNS Lymphoma. Diagnosis and treatment of primary CNS lymphoma in immunocompetent patients: guidelines from the European Association for Neuro-Oncology. Lancet Oncol. 2015;16(7):e322-e32.

36. Illerhaus G, Marks R, Ihorst G, Guttenberger R, Ostertag C, Derigs Gn, et al. High-dose chemotherapy with autologous stem-cell transplantation and hyperfractionated radiotherapy as first-line treatment of primary CNS lymphoma. J Clin Oncol. 2006;24(24):3865-70.

37. Alimohamed N, Daly A, Owen C, Duggan P, Stewart DA. Upfront thiotepa, busulfan, cyclophosphamide, and autologous stem cell transplantation for primary CNS lymphoma: a single centre experience. Leuk Lymphoma. 2012;53(5):862-7.

38. Del Rio MS, Ricard D, Houillier C, Navarro S, Gonzalez-Aguilar A, Idbaih A, et al. Prophylactic intrathecal chemotherapy in primary CNS lymphoma. J Neurooncol. 2012;106(1):143-6.

39. Ferreri A, Reni M, Pasini F, Calderoni A, Tirelli U, Pivnik A, et al. A multicenter study of treatment of primary CNS lymphoma. Neurology. 2002;58(10):1513-20.

40. Khan RB, Shi W, Thaler HT, DeAngelis LM, Abrey LE. Is intrathecal methotrexate necessary in the treatment of primary CNS lymphoma? J Neurooncol. 2002;58(2):175-8.

41. Pels $H$, Juergens $A$, Glasmacher $A$, Schulz $H$, Engert $A$, Linnebank $M$, et al. Early relapses in primary CNS lymphoma after response to polychemotherapy without intraventricular treatment: results of a phase II study. J Neurooncol. 2009;91(3):299-305.

42. Pels H, Schmidt-Wolf IG, Glasmacher A, Schulz H, Engert A, Diehl V, et al. Primary central nervous system lymphoma: results of a pilot and phase II study of systemic and intraventricular chemotherapy with deferred radiotherapy. J Clin Oncol. 2003;21(24):4489-95.

43. Omuro AMP, Chinot OL, Taillandier L, Ghesquieres $H$, Soussain C, Delwail $\mathrm{V}$, et al. Multicenter randomized phase II trial of methotrexate (MTX) and temozolomide (TMZ) versus MTX, procarbazine, vincristine, and cytarabine for primary CNS lymphoma (PCNSL) in the elderly: An Anocef and Goelams Intergroup study. American Society of Clinical Oncology; 2013.

44. Filley CM, Kleinschmidt-DeMasters B. Toxic leukoencephalopathy. N Engl J Med. 2001;345(6):425-32. 